Revue internationale de l'économie sociale

Recma

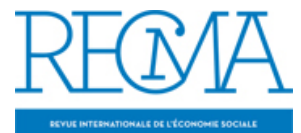

\title{
En hommage à Claude Vienney (1929-2001)
}

\section{Marie-Claire Malo et Marie Bouchard}

Numéro 283, février 2002

URI : https://id.erudit.org/iderudit/1022799ar

DOI : https://doi.org/10.7202/1022799ar

Aller au sommaire du numéro

\section{Éditeur(s)}

Institut de l'économie sociale (IES)

\section{ISSN}

1626-1682 (imprimé)

2261-2599 (numérique)

Découvrir la revue

\section{Citer ce document}

Malo, M.-C. \& Bouchard, M. (2002). En hommage à Claude Vienney (1929-2001). Revue internationale de l'économie sociale, (283), 5-14.

https://doi.org/10.7202/1022799ar d'utilisation que vous pouvez consulter en ligne.

https://apropos.erudit.org/fr/usagers/politique-dutilisation/ 


\title{
EN HOMMAGE À CLAUDE VIENNEY $(1929-2001)^{*}$
}

\author{
par Marie-Claire Malo et Marie Bouchard
}

\begin{abstract}
* Texte publié conjointement par Economie et Solidarités (revue du Ciriec-Canada) et la Revue internationale de l'économie sociale (Recma).
\end{abstract}

(1) Dans leurs recherches de mémoire de maîtrise ou de thèse de doctorat, des personnes telles que Gérard Allard, Michèle Blain, Michel Belley, Marie Bouchard, Alain Côté, Nicole Giroux, MarieClaire Malo, Claude Ouellet, Yves Paque, Yvan Rousseau, Joseph Yvon Thériault, Benoît Tremblay, Martine Vézina, Paul Vineen et bien d'autres se sont toutes inspirées de l'approche de Vienney, dans une plus ou moins large mesure.

(2) II s'agit du projet de recherche intégré France-Québec, coprésidé par Claude Vienney et Jean-Guy Desforges (alors président du Ciriec-Canada) et portant sur "L'émergence, le développement et la gestion des coopératives en France et au Québec ". Claude Vienney a séjourné au Québec à diverses reprises, invité notamment par le Ciriec-Canada, le centre de gestion des coopératives (Ecole des hautes études commerciales de Montréal), la chaire de coopération Guy-Bernier de I'université du Québec à Montréal, I'université du Québec à Chicoutimi, sans oublier I'Institut de recherche et d'enseignement pour les coopératives de l'université de Sherbrooke (Irecus) qui lui décerna un doctorat honoris causa.
$\mathrm{L}$

e jeudi 12 juillet 2001, Claude Vienney nous quittait, emporté par un cancer. C'est avec beaucoup de tristesse que tous ceux et celles qui l'ont connu ont appris son décès.

Claude Vienney est l'auteur de ce qui constitue certainement à ce jour l'une des synthèses les plus complètes pour l'analyse de l'organisation coopérative et de l'économie sociale. Ses ouvrages, notamment Socioéconomie des organisations coopératives, ont marqué et influencé de nombreux travaux de recherche dans le monde francophone, particulièrement au Québec ${ }^{(1)}$.

Mais, au-delà de son influence au plan conceptuel, Claude Vienney est aussi un mentor qui a été du parcours d'initiation et d'apprentissage de nombreux étudiants et praticiens de la coopération au Québec. On ne saurait dissocier l'approche de l'homme lui-même, celui qui a rassemblé, l'individu génératif qu'une, voire deux générations de chercheurs ont pris pour modèle. Claude Vienney nous a en effet impressionnés à plus d'un titre. D'abord, par ses textes si contrastants dans un univers d'écrits sur les coopératives qui, pour une large part, nourrissaient surtout nos projets, voire nos utopies. Lire Claude Vienney, outre qu'il s'agit d'un exercice que plusieurs qualifient de "gymnastique ", n'en demeure pas moins une révélation: il démontre la possibilité de porter des observations systématiques sur les pratiques coopératives, tout en se référant à une approche théorique rigoureuse qui ne classe pas une partie des observations hors champ pour cause d'écart à la " doctrine " coopérative. M. Vienney impressionne aussi par ses propres comportements. Illustrons ceci en rapportant le contexte dans lequel certains d'entre nous ont fait sa connaissance, alors qu' un groupe de Français participait à une rencontre où il allait être question de l'analyse comparée France-Québec de différents secteurs coopératifs ${ }^{(2)}$. Parmi tous ceux assis autour de la table, incontestablement le maître était cet homme très droit sur sa chaise, très concentré, un crayon à la main, gomme à effacer et blague à tabac à ses côtés, prenant des notes fines sur une feuille de papier divisée en deux colonnes. Il écoutait! Cette capacité d'écoute et sa grande humilité contrastaient avec l'impressionnante contribution que son œuvre allait laisser.

C'est donc cette personnalité qui a aussi certainement conduit tant de Québécois à adopter et à transmettre à leurs étudiants l'approche de la socio-économie de Claude Vienney. 


\section{Eléments de biographie ${ }^{(3)}$}

(3) D'après l'« Entretien avec Claude Vienney " réalisé par André Chomel et Marie-Claire Malo les 16 et 20 février 1996 à Paris. Cet article sera publié conjointement par la chaire de coopération Guy-Bernier de I'Uqam et la Revue internationale de l'économie sociale (Recma).
Né en 1929 à Paris, Claude Vienney est fier d'être un Parisien de quatrième génération. Fils de pasteur protestant, il participe au mouvement du christianisme social, qui offre chaque été un camp de formation à des jeunes. C'est là qu'il rencontre Georges Lasserre, qui sera aussi son professeur. Claude Vienney entreprend des études dans le but de devenir juge d'enfants sous l'influence d'un oncle, avocat du Parti communiste français. Il aime beaucoup les enfants, il en aura cinq, mais ne deviendra jamais juge d'enfants! Le contenu économique des études de droit qu'il poursuit l'intéresse au plus haut point. Et dans le cours de Lasserre, c'est la partie sur l'histoire du syndicalisme qu'il trouve la plus intéressante.

Chez les étudiants de sa génération, on ne retrouve pas grand intérêt pour les coopératives. Ce n'est donc pas sur les coopératives que Claude Vienney veut initialement faire sa thèse, mais plutôt sur le comportement du consommateur. Un jour, son professeur le met en contact avec la Société générale des coopératives de consommation (SGCC). Le secrétaire de rédaction de la revue Coopération vient de partir, recruté par la Fédération nationale des coopératives de consommation (FNCC). Le poste de secrétaire de rédaction est un emploi à mi-temps qui convient très bien à un étudiant de doctorat. Fort de cette nouvelle expérience, Claude Vienney décide de réaliser sa thèse sur les coopératives, puisqu'il travaille déjà dans le domaine. Il entreprend de ne produire rien de moins que la comptabilité nationale du secteur coopératif! "C'était dur » avoue-t-il, car il n'est " pas tellement statisticien ". Si bien qu'après quelques semaines il fait ce que plus tard il conseillera à ses étudiants: "Vient un moment où il faut trancher et faire avec ce qu'on a quel que soit son objectif de thèse! "Il fait donc une thèse en deux parties: une première, plus étoffée, sur l'histoire de la pensée économique en rapport avec la doctrine coopérative, et une deuxième sur la comptabilité nationale dans laquelle, en conclusion, il propose une politique de développement coopératif comprenant notamment une politique d'éducation. Il manifeste en effet un intérêt pour l'éducation populaire qui lui vient de son travail à la fois au sein du christianisme social et à l'association Peuple et Culture.

De sa thèse, André Chomel dit qu'elle déclenche un séisme! Claude Vienney préfere dire qu'à la Société générale des coopératives de consommation ses collègues pensent plutôt qu'il a enfoncé une porte ouverte, en disant que les coopératives de consommateurs ne peuvent pas envahir toute l'économie! "J'ai critiqué ouvertement le fameux syllogisme de Gide, précise Vienney. J'ai fait une étude soulignant au moins deux raisons de critiquer la construction théorique de Gide sur la république coopérative des consommateurs. " La première, c'est qu'à partir du moment où les consommateurs ne font pas partie de la même classe sociale ils ne sont pas du tout solidaires. La deuxième raison commande de faire ressortir l'intervention de l'Etat dans l'économie, car Vienney est déjà keynésien - et il le restera. 
Ces deux aspects n'ont pas plu dans les milieux coopératifs de consommation. Bugnon, un militant des coopérateurs de Lorraine, dira: "Vous avez détruit les idoles, nos dieux, notre religion, soubaitons que vous fassiez une nouvelle religion, une nouvelle cathédrale! » Mais il reçoit aussi des félicitations, notamment de Lacour, alors président du Crédit coopératif français, ainsi que de Chomel, le numéro deux à la Caisse centrale du Crédit coopératif (CCCC), qui cherche alors une stratégie pour sortir le Crédit coopératif du champ exclusif des coopératives de consommateurs et des sociétés coopératives ouvrières de production (Scop).

Orsini, le patron de Vienney à la revue des coopératives de consommation, est venu le voir un jour pour lui annoncer la mort de Georges Fauquet. Orsini lui remet un excellent texte d'Hirschfeld sur Fauquet, que Vienney publie dans la revue Coopération. C'est la première fois qu'il entend parler de Fauquet. Et c'est Fauquet, et non Gide, qui influencera Vienney: «Avec Gide, nous dit Vienney, on n'a pas d'outils, alors qu'avec ce quécrit Fauquet on peut travailler toute sa vie, et pourtant il a beaucoup moins écrit que Gide. "

Secrétaire de rédaction durant une dizaine d'années, de 1953 à 1962, Vienney redore le blason de la revue en obtenant des contributions prestigieuses dont celle de l'économiste Marshall. Il quitte son poste lorsque la revue atteint un nouveau plafond. Son patron, pourtant, ne demande pas mieux que de le garder à la Société générale des coopératives de consommation et de le faire travailler aux services techniques des coopératives de consommateurs. Mais Vienney préfere devenir directeur de stages à l'Institut des sciences sociales du travail (ISST).

Marcel David, le directeur de l'ISST, conseillé par Henri Desroche, recrute Claude Vienney pour travailler à la formation des responsables syndicaux. "C'est prenant, c'est extraordinaire ", nous dit Vienney, qui aimera travailler avec des adultes engagés. Il rappelle que pendant le mouvement de mai 1968, à la section d'éducation ouvrière de l'ISST, on s'est mis à discuter d'autogestion: "Ce que le patron David et les syndicats qui étaient au conseil d'administration de l'ISST ont très mal pris! "Vienney et ses copains sont en effet à l'origine de la création d'une section syndicale à l'ISST. Une initiative considérée comme scandaleuse, mentionne Vienney. En entrant à 'ISST, Claude Vienney fait officiellement partie de la fonction publique, de sorte qu'en le quittant, en 1969, il peut entrer à l'UR12 de l'université de Paris-I. Militant syndical, il continuera son action à Paris-I, en participant à la grande bataille sur le statut des assistants.

Faut-il voir, dans ce passé syndical, une source d'influence sur les réflexions de Vienney quant aux voies de rééquilibrage de la combinaison caractéristique des coopératives? Vienney ne met-il pas en évidence les acteurs collectifs, par exemple les groupes de défense des consommateurs dans le

(4) Claude Vienney (1980), " Rapport d'activités et rapports de sociétariat ", in Jean-Guy Desforges et Claude Vienney (dir.), Stratégie et organisation de l'entreprise coopérative, Montréal, Editions du jour et Ciem, p. 251-283. cas des coopératives de consommation ${ }^{(4)}$ ? Si l'on peut y voir un rapport aux associations à caractère syndical, Vienney, lui, y voit plutôt une réaction à son atavisme protestant: "Les protestants sont tellement individualistes quil ne me déplaisait pas de dire que l'individu, c'est bien joli, mais que dans une entreprise, ce n'est pas l'individu travailleur qui discute les clauses 
avec le patron! Alors on peut dire qu'effectivement j'ai eu un intérêt équivalent pour les syndicats et pour les coopératives. C'est d'ailleurs pour cela que jétais intéressé à faire de la formation syndicale et même, à l'origine, quand jai quitté les coopératives de consommation pour travailler avec les syndicats, j'avais plutôt l'impression d'une promotion du point de vue de mes intérêts. J'avais plutôt l'impression de travailler avec des groupes plus actifs, plus dans l'ordre de la discussion collective de tous les problèmes contemporains. "

Tout en travaillant à l'ISST, Vienney entreprend une activité de recherche et d'enseignement sur les coopératives, au Collège coopératif de Paris dirigé par Henri Desroche. "J'ai gardé un souvenir très précis de la première fois que j'ai vu Desroche. J'étais en train de terminer ma thèse, se rappelle Vienney. Je travaillais à la bibliothèque de l'Entente communautaire. J'étais en train de travailler à une table, et puis j'ai senti quelqu'un, j'ai senti qu'il y avait quelqu'un derrière moi. J'ai continué à travailler, et puis ce quelqu'un derrière moi est resté longtemps, longtemps sans rien dire. Alors au bout d'un moment, je me suis retourné et c'était effectivement Desroche. C'était en 1958 ou 1959. Desroche travaillait au CNRS à l'époque. "

Le rôle qu'a alors joué Desroche, nous confie Vienney, "c'est de totalement revitaliser un champ de recherche qui était mort. Lasserre était un excellent enseignant, mais disons que ce n'était pas un chercheur. S'il n'y avait pas eu Desroche, aucun jeune de mon âge ne serait entré dans ce champ! Ce n'est pas tellement à cause de ce que Desroche disait qu'on y est entré, car on n'était pas forcément d'accord avec ce qu'il disait, mais c'est parce qu'il a revitalisé, en quelque sorte il a reprouvé qu'il y avait encore des choses à chercher là. " Desroche, Vienney et Denise Rocher sont les trois signataires de la déclaration d'association du collège coopératif de Paris. Vienney poursuit: "Une des idées de génie de Desroche, dans sa vie, fut de sapercevoir qu'il y avait un créneau: des étudiants africains venaient à Paris en ayant dans leur centre d'intérêt d'étudier les coopératives, mais il n'y avait pas pour eux d'accès à un diplôme. C'est l'origine de la combinaison entre le collège coopératif et le centre de recherches coopératives de l'Ecole des hautes études en sciences sociales (EHESS), où Henri Desroche était directeur d'étude. L'originalité et la force étaient de combiner une association et un centre de recherche universitaire."

C'est durant la période de 1969 à 1982 que Claude Vienney travaille de façon plus systématique et plus complète comme universitaire à la fois à l'université de Paris-I et au centre de recherches coopératives de l'EHESS. "Cette période la plus complète correspond à mon métier d'universitaire et donc d'enseignant-chercheur, précise Vienney. A Paris-I, je n'étais qu'enseignant et il ne m'était jamais venu à l'esprit d'y créer un centre de recherche, puisqu'ily en avait un à l'Ecole des hautes études et que jy étais associé. "

Dans la période postérieure, c'est-à-dire de 1982 jusqu’à sa retraite en 1990, Vienney ne travaillera plus qu'à Paris-I. En effet, avec la retraite de Desroche, le centre de recherches coopératives de l'EHESS a disparu, même si Desroche avait envisagé, à une certaine époque, que Maxime Haubert le remplace comme directeur d'étude à l'école et que Claude Vienney lui succède comme directeur du collège. 
(5) Au Canada, en langue française, Benoît Lévesque, Marie-Claire Malo et Michelle Rhéaume-Champagne ont réalisé une base de données bibliographiques similaire, sur les coopératives et autres entreprises d'économie sociale, couvran la période 1975-2000. Pour consultation, s'adresser à michelle.champagne@hec.ca.
Nous sommes donc en 1982: l'économie sociale est à la mode en France. L'université de Paris-I lance son diplôme d'études supérieures en économie sociale et l'initiatrice du projet demande à Claude Vienney, le spécialiste, de s'en occuper. D'abord hésitant parce qu'il n'est pas un fanatique de l'économie sociale, Vienney accepte finalement, la fin des activités au collège lui laissant plus de temps libre. Mais il a d'autres raisons. D'abord, il veut revenir à son activité de départ: la formation d'adultes. Même s'il ne l'a jamais vraiment abandonnée, il veut vraiment qu'elle occupe une portion plus importante de son travail. Il a auparavant appris, à Peuple et Culture, la méthode d'entraînement mental, le professionnalisme en formation d'adultes. Vienney précise que, s'il doit quelque chose à Desroche, c'est incontestablement d'avoir pris conscience de tout ce que l'on peut tirer des projets de recherche. De plus, ajoute Vienney, "javavis besoin de prouver à Desroche et à moi-même que l'on pouvait faire ça, à Paris-I. " Alors que Desroche disait des relations avec les universités: "La greffe ne prend pas, elle ne peut pas prendre ", Vienney voulait prouver que cela était possible. "Et donc, dans le diplôme en économie sociale, on faisait des cours la première année, ça convenait très bien à Paris I, mais la deuxième année, on faisait une recherche personnelle."

A la même époque, Vienney crée la banque de travail inter-universitaire pour la documentation des programmes de recherche et d'enseignement sur l'économie sociale: la BTI. C'est d'abord pour récupérer le centre de documentation inter-coopératives et les services de Jacqueline Soyez, au moment où le mouvement coopératif abandonne l'activité. C'est aussi une réaction d'autoorganisation pour des professeurs comme Vienney, Demoustier, Mevelec, Nicolas, Parodi et d'autres appelés à donner une formation en économie sociale. Vienney, alors en avance de dix ans, veut pousser plus loin le projet, l'informatiser en collaboration avec la Sydes ${ }^{(5)}$. Toute une série d'obstacles feront avorter le projet, même si l'initiative est tout à fait louable et même si Michel Rocard, alors ministre du Plan et ex-camarade de classe de Vienney avec qui il a fraternisé, accorde une subvention en appui au projet. Pourtant le réseau BTI joue un rôle dans le projet intégré France-Québec portant sur les coopératives, projet coordonné par le Ciriec-Canada, alors présidé par Jean-Guy Desforges, professeur aux HEC de Montréal, et par la BTI de Claude Vienney. «On a raflé toutes les bourses des programmes France-Québec, c'était formidable " se souvient Vienney souriant.

Mais la BTI n'a pas toute la portée que l'on en escomptait, notamment parce que les cours en économie sociale ne se multiplient pas à travers la France. Même à Paris-I, le diplôme est abandonné faute de participants. Il faut dire que c'est une formation de culture générale, et non pas une formation professionnelle, Vienney n'ayant jamais pensé que l'économie sociale était un champ disciplinaire. "Par conséquent, la formation était fragile, elle reposait sur un financement des participants beaucoup plus que des entreprises de l'économie sociale. "Vienney a bien fait la tournée des " grands ducs ", mais sans les convaincre. Il n'est peut-être pas assez agressif, lui diront ses collègues, en particulier Jean-Yves Manoa, qui lui succédera à Paris-I. 
A la même époque, des coopératives s'effondrent. Vienney, à la demande de Chomel, écrit dans la Recma, la Revue des études coopératives, mutualistes et associatives, une petite notice intitulée "Pour qui sonne le glas? " "J'ai vécu l'effondrement avec tristesse pour les amis que j'avais dans ce milieu, mais pas pour le mouvement, précise Vienney. On l'attendait depuis si longtemps [...]. Je veux dire que le mouvement, comme mouvement, avait quand même perdu de sa vitalité. Disons que je l'ai ressenti intellectuellement quand même, comme la fin d'une histoire. "Cette évolution, Vienney l'a pourtant pressentie à travers sa découverte des « lois » de formation et transformation des coopératives.

\section{Emergence et transformation des organisations}

(6) Claude Vienney (1992-1993) "L'analyse socio-économique des coopératives justifie-t-elle la recherche d'instruments de gestion spécifiques", Coopératives et Développement, Revue du CiriecCanada, vol. 24, $n^{\circ} 1$, p. 10.
Claude Vienney partage avec son prédécesseur Georges Fauquet la même vision du développement coopératif (sous-système et non pas système comme le souhaitaient Gide et, au Québec, Angers) et la même définition de la coopérative: combinaison d'un groupement de personnes et d'une entreprise. Vienney rejette la notion d'esprit coopératif: "Qu'est-ce que c'est? " dit-il en boutade. Dans la coopérative, il précise que les modes de raisonnement spécifiques sont déterminés par la double place des membres en rapport de sociétariat (propriété, mais aussi adhésion) et en rapport d'activités (producteurs-fournisseurs, travailleurs-salariés, consommateursclients) avec l'entreprise.

Vienney réexplique l'émergence de la forme coopérative en faisant ressortir son double rôle d'agent d'adaptation des activités des membres aux règles de l'économie de marché et d'agent de transformation des membres en les faisant accéder collectivement au pouvoir de l'entrepreneur. Pour l'économiste qu'il est, l'entrepreneur est l'agent économique qui a le pouvoir sur la combinaison productive qu'est l'entreprise et sur l'affectation des excédents ou surplus. Qui est l'entrepreneur? Dans le système capitaliste, c'est l'investisseur. Dans le sous-système coopératif, c'est le groupement de personnes en rapport d'activités qui a le contrôle sur les orientations de l'entreprise. Vienney explique que les personnes qui constituent le sociétariat à l'émergence des coopératives appartiennent à des catégories relativement dominées. Les membres à l'origine, dit-il, ne sont " ni trop riches ni trop pauvres". Il montre aussi que les coopératives prennent place dans des secteurs ou segments relativement peu rentables et donc délaissés par l'entrepreneur capitaliste.

Vienney fait ressortir la cohérence des quatre règles caractérisant respectivement ${ }^{(6)}$ :

- le groupement de personnes (égalité des personnes, " une personne égale un et un seul vote ");

- l'entreprise (propriété collective des résultats réinvestis);

- la mise en rapport des personnes avec l'entreprise (rapport d'activités comme membres-fournisseurs, membres-travailleurs, membres-clients); 
(7) Claude Vienney (1994), L'économie sociale, Paris, La Découverte, "Repères ", p. 71.

(8) Marie-Claire Malo (1981-1982) «Entretien avec Claude Vienney sur Socio-économie des organisations coopératives ", Revue du CiriecCanada, vol. $14, n^{\circ} 1$, p. 163.

(9) Claude Vienney (1994), L'économie sociale, Paris, La Découverte, "Repères ", p. 10.
- la mise en rapport de l'entreprise avec le groupement de personnes sur l'entreprise (une fois la réserve constituée, les excédents qui restent sont partagés au prorata des transactions du membre avec sa coopérative: ventes, salaires, achats).

Il approfondit l'impact d'un fonctionnement comme sous-système dans un système aux règles du jeu différentes. Si, à l'origine, les orientations stratégiques de l'entreprise sont déterminées par le groupement de personnes (détermination génétique), en fonctionnant en économie de marché, il arrive un point de retournement où elles sont choisies selon les critères du marché (détermination fonctionnaliste). La coopérative en vient même à sélectionner ses membres selon les règles du jeu dans son secteur. Plutôt que conclure à la dénaturation, Vienney y voit une transformation.

La pièce maîtresse de son œuvre, présentée dans Socio-économie des organisations coopératives et reprise, en l'appliquant aux mutuelles et aux associations gestionnaires, dans L'économie sociale, est sans contredit son dispositif d'analyse de la "correspondance entre les règles de certaines institutions, la place de leurs activités dans l'économie et l'identité des acteurs qui en sont les associés participants ${ }^{(7)}$ ". Parlant de ce dispositif, Vienney, toujours trop modeste, ramène son apport original à "une certaine obstination à représenter les différentes activités et à caractériser les acteurs toujours de la même manière, d'une manière méthodologique, en utilisant pour cela des apports théoriques déjà existants, mais seulement comme des instruments". L'originalité de Vienney aura justement été de combiner deux courants de pensée apparemment opposés: "Je suis en effet assez hérétique, car je prétends qu'il faut utiliser à la fois le modèle fonctionnaliste de Walras et le modèle transformationniste de Marx pour comprendre ces organisations ${ }^{(8)}$. "

Si Vienney, combinant ces deux approches, a magistralement expliqué l'émergence de la forme coopérative alors que le capitalisme se développe au XIX ${ }^{e}$ siècle, son œuvre rejoint aussi les préoccupations contemporaines, car il a expliqué les « lois » de transformation des coopératives. Il démontre que les problèmes rencontrés par les grandes organisations coopératives et mutuelles d'un certain âge "viennent de ce qu'elles se sont beaucoup transformées depuis leurs origines. Leurs activités ne sont plus délaissées par, mais en concurrence avec celles des entreprises de type capitaliste. Les pouvoirs publics les incitent à se procurer sur le marché les ressources dont elles ont besoin plus qu'ils n'apportent d'aide à leur financement. Leurs statuts ont été renégociés pour trouver un compromis avec des financiers non participants à leurs activités ${ }^{(9)}$. " Il est certain que les coopératives se sont transformées, nous dit Vienney, mais contrairement à Oppenheimer, il ne conclut pas nécessairement à leur dénaturation: "Ce que je pense, c'est que ce n'est pas une dénaturation, mais c'est quand même une transformation. Et si j'ai pensé que ce n'était pas une dénaturation, c'est que, quand les coopératives se sont formées comme organisations ayant leurs caractères de coopérative, c'était déjà le produit d'une transformation par rapport aux communautés des origines. Autrement dit, ce n'était plus des communautés, c'était devenu des coopératives, et ça, c'est donc une transformation. Et pourquoi parler de dénaturation? Dans les 
sciences sociales, on ne doit pas parler de dénaturation, parce que cela supposerait que les organisations ont une sorte de statut naturel, qu'elles seraient conformes à la nature. Ce qui n'est pas mon sentiment. "

Il restait donc à chercher les forces de rééquilibrage pouvant conduire à un retour de l'ajustement mutuel du groupement de personnes et de l'entreprise. Vienney nous confie: "Il fallait tout de même en arriver à formaliser une régulation, c'est-à-dire une transformation qui aboutit effectivement à changer un certain nombre de caractéristiques de l'organisation, mais qui la ramène malgré tout à des caractéristiques de ses origines". Or, "jai toujours pensé, c'est peut-être le résultat de ma formation d'économie politique classique, que la caractéristique d'origine, c'est ce que j'appelle la préférence pour l'activité par rapport à la rentabilité. "C'est justement cette double détermination, associative et entrepreneuriale, qui maintient la combinaison coopérative, que les forces centrifuges de l'environnement économique tendent à dissocier.

Son œuvre est d'une intense actualité alors que se redynamisent et se recomposent les rapports entre sociétés de personnes: associations, coopératives, mutuelles et syndicats.

\section{Une profession et une vocation}

En terminant, laissons encore la parole à Claude Vienney qui, le jour de son départ à la retraite, avait choisi, en réponse à ses collègues qui lui rendaient hommage, le thème de la Berufde Marx Weber, à propos du protestantisme et de l'esprit du capitalisme: "Le terme a deux sens: la Beruf, c'est une profession et une vocation. Et effectivement, ces fameux protestants, ils étaient entrepreneurs par vocation et par profession. J'ai toujours été pris par ces deux pôles: la vocation et la profession. Par vocation, j'ai fait de la formation d'adultes et je me suis intéressé aux coopératives, mais aussi par profession. Et jai toujours fait attention de ne pas me laisser entraîner par ma vocation. Toujours pour la même raison: quand vous dites des choses à des étudiants, vous ne développez pas des idées personnelles. Je n'ai jamais eu l'idée que je développais des préférences personnelles sur les coopératives. Il y avait aussi une contrainte professionnelle: que ce que je disais était vrai et était juste. Autrement dit, quelque chose peut être vrai et peut ne pas être conforme à la justice. J'ai essayé de travailler dans un champ où l'on peut argumenter sur ces deux critères: la vérité et la justice."

Voilà donc aussi à quoi nous convie Vienney, tout autant par le caractère attachant de sa personne que par le caractère stimulant et pertinent de ses écrits. 


\section{Bibliographie partielle de documents disponibles à la bibliothèque des HEC (1)}

Vienney, Claude (1997), « Le maintien et le renforcement de la réciprocité entre l'entreprise et le mouvement ", in Benoît Lévesque (dir.), Desjardins: une entreprise et un mouvement? Sainte-Foy, Presses de l'université du Québec, p. 323-328.

Vienney, Claude (1994), L'économie sociale, Paris, Editions La Découverte, "Repères ", $125 \mathrm{p}$.

Vienney, Claude (1992-1993), «L'analyse socioéconomique des coopératives justifie-t-elle la recherche d'instruments de gestion spécifiques ", Coopératives et Développement, Revue du CiriecCanada, vol. 24, no 1, p. 5-15.

Vienney, Claude (1990), " Y a-t-il place pour les coopératives dans les institutions du logement social? ", communication présentée au colloque de l'Addes « L'économie sociale en évolution: transformation des organismes, maintien de leur identité? " le 13 mars 1990, Paris, Association pour le développement de la documentation sur l'économie sociale, $33 \mathrm{p}$.

Vienney, Claude (1989?), Fonctionnement et financement des coopératives d'habitation en Europe, Paris, Comité européen de coordination de l'habitat social (pagination multiple).

Vienney, Claude (1989), " Les rapports entre les coopératives et l'Etat ", in Bernard Denault (dir.), Les relations Etat-coopératives, actes du colloque international organisé par l'Institut de recherche et d'enseignement pour les coopératives, Irecus, en collaboration avec le Conseil canadien de la coopération et la Cooperative Union of Canada, et tenu à Sherbrooke du 31 mai au 4 juin 1987, Sherbrooke, Québec, Institut de recherche et d'enseignement pour les coopératives de l'université de Sherbrooke, p. 25-58.

Vienney, Claude (1986), Les organismes de l'économie sociale renforcent-ils leur identité dans la période contemporaine? colloque « Economie sociale et financements publics ", 17 juin 1986,
Nanterre, Association pour le développement de la documentation sur l'économie sociale, $38 \mathrm{p}$. Vienney, Claude (1986), Gestion, financement et développement des coopératives d'habitation, Colloque international « Emergence, développement et gestion des coopératives en France et au Québec ", tenu du 26 au 29 mai 1986, Montréal, Ciriec-BTI, 35 p.

Vienney, Claude et Jean-Guy Desforges (dir.) (1986), Emergence, développement et gestion des coopératives en France et au Québec: résumés des communications, colloque international «Emergence, développement et gestion des coopératives en France et au Québec ", tenu du 26 au 29 mai 1986 à l'Ecole des hautes études commerciales de Montréal, Montréal, Centre interuniversitaire de recherche, d'information et d'enseignement sur les coopératives (Ciriec), $70 \mathrm{p}$.

Nicolas, Philippe et Claude Vienney (1985), Emploi et coopératives d'entrepreneurs individuels: les cas des coopératives d'agriculteurs commerçants et artisans, Nanterre, Association pour le développement de la documentation sur l'économie sociale, $98 \mathrm{p}$.

Vienney, Claude, Alain Prigent, Roland Charbonneau et Jean-Pierre Deslauriers, avec la participation de Paul Prévost (1985), Analyse socio-économique comparée des coopératives d'habitation en France et au Québec: rapport de recherche, Chicoutimi, Groupe de recherche et d'intervention régionales (GRIR), université du Québec à Chicoutimi, 308 p.

Vienney, Claude (1983), « Concepts et champs de l'économie sociale, conditions et enjeux d'une information statistique ", in La délimitation et l'organisation du champ statistique de l'économie sociale, Nanterre, Association pour le développement de la documentation sur l'économie sociale, p. 4-21.

Vienney, Claude (1983), Les activités, les acteurs et les riegles des organismes de l'économie sociale (coopératives, mutuelles, associations gestionnaires), 
Savignac-sur-Orge, France, Banque de travail interuniversitaire pour la documentation des programmes d'enseignement et de recherches en économie sociale, $37 \mathrm{p}$.

Vienney, Claude (1983?), Approches de l'économie sociale, Savignac-sur-Orge, France, Banque de travail interuniversitaire pour la documentation des programmes d'enseignement et de recherches en économie sociale, $36 \mathrm{p}$.

Vienney, Claude (1980-1982), Socio-économie des organisations coopératives, tome 1, Formation et transformations des institutions du secteur coopératif français, tome 2, Analyse comparée des coopératives fonctionnant dans des systèmes socio-économiques, Paris, Coopérative d'information et d'édition mutualiste, "Tiers-secteur ". Desforges, Jean-Guy et Claude Vienney (1980), Stratégie et organisation de l'entreprise coopérative, textes présentés lors du séminaire de l'université coopérative internationale tenu à Montréal en 1978, Montréal, Editions du jour, 424 p.

Vienney, Claude (1980), " Rapport d'activités et rapports de sociétariat ", in Jean-Guy Desforges et Claude Vienney, Stratégie et organisation de l'entreprise coopérative, Montréal, Editions du jour et Ciem, p. 251-283.

Vienney, Claude (1980), "Les coopératives et le développement socio-économique ", in Spécificité de l'entreprise et de la gestion des coopératives, Montréal, Ecole des hautes études commerciales, centre de gestion des coopératives, cahier T-80-13, p. 10-27.

Vienney, Claude (1980), Initiation à l'économie politique par l'analyse de texte, vol. 1, La formation de l'homme économique, d'après La Force des forts de J. London, vol. 2, La formation du capitalisme marchand, d'après Le Médecin de campagne de H. de Balzac, Gujan-Mestras, France, Intermedia.

Melançon, Jacinthe, avec la collab. de JeanGuy Desforges et Claude Vienney (1978), "Notes sur la "session synthèse" du ler septembre 1978 ", in Séminaire université coopérative internationale, La stratégie et l'organisation de l'entreprise coopérative, Montréal, Ecole des hautes études commerciales, Centre de gestion des coopératives, $25 \mathrm{p}$.

Vienney, Claude (1976-1977), Les institutions coopératives comme agents de transformations économiques et sociales, Paris, université Paris-I, UER Travail et questions sociales, Ecole des hautes études en sciences sociales, centre de recherches coopératives, $74 \mathrm{p}$.

Desroche, Henri et Claude Vienney, avec la collab. d'André Chomel et al. (1970), Epargne et crédit en développement coopératif, Paris, Union du crédit coopératif, $226 \mathrm{p}$.

Vienney, Claude (1966), L'économie du secteur coopératiffrançais, Paris, Editions Cujas, "Théories et pratiques de sciences économiques ", $460 \mathrm{p}$.

Vienney, Claude (1961), Vers une analyse économique du secteur coopératif, Paris, Bureau d'études coopératives communautaires, $204 \mathrm{p}$.

(1) Consultation du catalogue HECtor sur le site Internet http://www.hec.ca/biblio. 